\title{
Factors affecting particle collection by electro-osmosis in microfluidic systems
}

\begin{abstract}
Alternating-current electro-osmosis, a phenomenon of fluid transport due to the interaction between an electrical double layer and a tangential electric field, has been used both for inducing fluid movement and for the concentration of particles suspended in the fluid. This offers many advantages over other phenomena used to trap particles, such as placing particles at an electrode centre rather than an edge; benefits of scale, where electrodes hundreds of micrometers across can trap particles from the molecules to cells at the same rate; and a trapping volume limited by the vortex height, a phenomenon thus far unstudied. In this paper, the collection of particles due to alternating-current electro-osmosis driven collection is examined for a range of particle concentrations, inter-electrode gap widths, chamber heights and media viscosity and density. A model of collection behaviour is described where particle collection over time is governed by two processes, one driven by the vortices and the other by sedimentation, allowing the determination of the maximum height of vortex-driven collection, but also indicates how trapping is limited by high particle concentrations and fluid velocities. The results also indicate that viscosity, rather than density, is a significant governing factor in determining the trapping behaviour of particles.
\end{abstract}

Keyword: ACEO; Concentration; Dielectrophoresis; Electrohydrodynamics; Electrokinetics 